\title{
The Tyrosine Phosphatase SHP2: A Key Molecule Linked both Type 2 Diabetes and Cancers?
}

\author{
Hu Luo, Chunlan Tang, Xuemei Yang and Xiangdong Zhou* \\ Department of Respiratory Medicine, the First Affiliated Hospital of Third Military Medical University, Chongqing 400038, P.R. China
}

\begin{abstract}
Emerging epidemiological evidence suggests that T2DM may be associated with an increased risk of certain cancers. However, the underlying molecular mechanism linked these two diseases remains largely unknown. SHP2, a non-receptor protein tyrosine phosphatase encoded by pro-oncogene PTPN11, has been reported involved in insulin resistance through PI3K/Akt/mOTR signaling and has also been considered to play a vital role in carcinogenesis via Ras/Erk pathways. Based on our previous studies, we hypothesize that SHP2 may present a key molecule linked both T2DM and cancers through both Ras/Erk and PI3K/AKT/mTOR signaling pathways. We believe that the comprehensive and detailed investigation of SHP2 may provide a new insight into the underlying molecular mechanism linked both T2DM and cancers, thereby facilitating the process to discover novel therapeutic targets to prevent and treat cancers.
\end{abstract}

\section{Keywords: Type 2 Diabetes Mellitus; Cancer; Breast oncogenesis}

\section{Background}

Type 2 Diabetes Mellitus (T2DM) is a common disorder, which is characterized by high blood glucose concentration in the context of insulin resistance and/or relative insulin deficiency. Increasing epidemiological evidence suggests that T2DM may be associated with an increased risk of certain cancers including pancreatic [1], hepatic [2], lung [3], colorectal [4], breast [5], bladder [6], gastric [7] and endometrial [8] cancers. These results suggest that the comprehensive investigation of the mechanisms responsible for DM to facilitate occurrence of cancer may provide us new potential therapeutic targets. However, the current studies have been mainly focused on the relationship between DM and cancers, and the molecular mechanism linked these two diseases remains largely unknown.

Abnormal protein tyrosine phosphorylation underlies various diseases of deregulated growth and differentiation, including cancer [9-11]. Src-homology 2 domain-containing phosphatase (SHP2) is a non-receptor protein tyrosine phosphatase encoded by the first identified proto-oncogene PTPN11. Genetic and biochemical studies in recent years have suggested that SHP2 plays a broad role not only in cell proliferation [12], survival [13], and differentiation [14], but also in development [15,16] and tumorigenesis [17-19] of malignancies via Ras/Erk [20], PI3K/Akt [21] and other signaling pathways. In accordance to a recent study [18], our previous studies [22-24] have demonstrated that SHP2 plays a crucial role in breast oncogenesis. Recently, we have also found for the first time that SHP2 is widely expressed by lung cancer cells, and that the high expression of SHP2 may promote the invasion and metastasis of NSCLC through angiogenesis and the lymphatic system $[25,26]$. As T2DM has also been considered as a risk factor of many kinds of cancers, we begin to speculate whether SHP2 plays a role in the pathogenesis of T2DM? If so, should SHP2 be a potential target linked both T2DM and cancers?

Under this speculation, we have performed a systematic search on the literature data base. It is generally accepted that insulin resistance is due to defective insulin signaling and thus results in the progression of T2DM, but details remain largely unknown. In recent years, several clinical studies [27-30] have declared that metformin, which is considered as a first-line treatment modality for T2DM, reduces incidence of neoplastic diseases in T2DM patients, as compared to other anti-diabetic agents. Studies [31,32] have showed that metformin inhibits the mammalian Target Of Rapamycin Complex 1 (mTORC1), a molecule downstream of PI3K/Akt, resulting in decreased cancer cell proliferation. These new encouraging experimental data support that the crucial involvement of PI3K/Akt/mTOR signaling pathway in the insulin resistance and progression of diabetes. Besides, Bifulco [33] showed that glucose regulated insulin signaling via the IRS1/MAPK pathway and mitogenesis by modulating the activity and subcellular localization of the SHP2 tyrosine phosphatase. Using a mouse model, Princen found that insulin resistance and impaired glucose uptake existed in SHP2-deficient mice [34]. Further studies [35-39] suggest that SHP2 recruited by Insulin Receptor Substrate-1 (IRS-1), acts as a signal coordinator in pancreatic beta-cells and controlled insulin biosynthesis to maintain glucose homeostasis through Akt and Erk pathways. These abovementioned results strongly suggest that SHP2, recruited by IRS1 , may present a key molecule involved in both T2DM and cancers via both Ras/Erk and PI3K/Akt/mTOR signaling pathways.

\section{The Hypothesis}

Epidemiological evidence suggests that T2DM is a risk factor of lung cancer. Studies focused on the common signal pathway shared by both T2DM and cancers may provide us new potential therapeutic targets. SHP2 plays a vital role in both insulin resistance and cancers through PI3K/Akt/mOTR and Ras/Erk pathways respectively. We speculate that SHP2 may present a key target linked both T2DM and cancers: on one hand, the high expression or activation of SHP2 results in excessive tyrosine dephosphorylation on IRS-1/2, which is a key part of insulin resistance. On the other hand, overactivation of SHP2 can sequentially activate PI3K/Akt/mTOR and Ras/Erk pathways, while both pathways are generally accepted to be closely associated with tumorigenesis and progression of cancers. The role of SHP2 involved in T2DM and cancers are schematically described in Figure 1. Further investigation of SHP2 may provide a new insight into the underlying molecular mechanism linked both T2DM and cancers, thus help

*Corresponding author: Zhou Xiangdong, Department of Respiratory Medicine The First Affiliated Hospital of Third Military Medical University, No.30 in Gao Tanyan Street, Sha Pingba Distinct, Chongqing, P.R. China, Tel: +86-023-687-55679; E-mail: xiangdongzhou@126.com

Received February 12, 2014; Accepted April 17, 2014; Published April 20, 2014

Citation: Luo H, Tang C, Yang X, Zhou X (2014) The Tyrosine Phosphatase SHP2 A Key Molecule Linked both Type 2 Diabetes and Cancers? Med chem 4: 435-438. doi:10.4172/2161-0444.1000175

Copyright: () 2014 Luo H, et al. This is an open-access article distributed under the terms of the Creative Commons Attribution License, which permits unrestricted use, distribution, and reproduction in any medium, provided the original author and source are credited. 


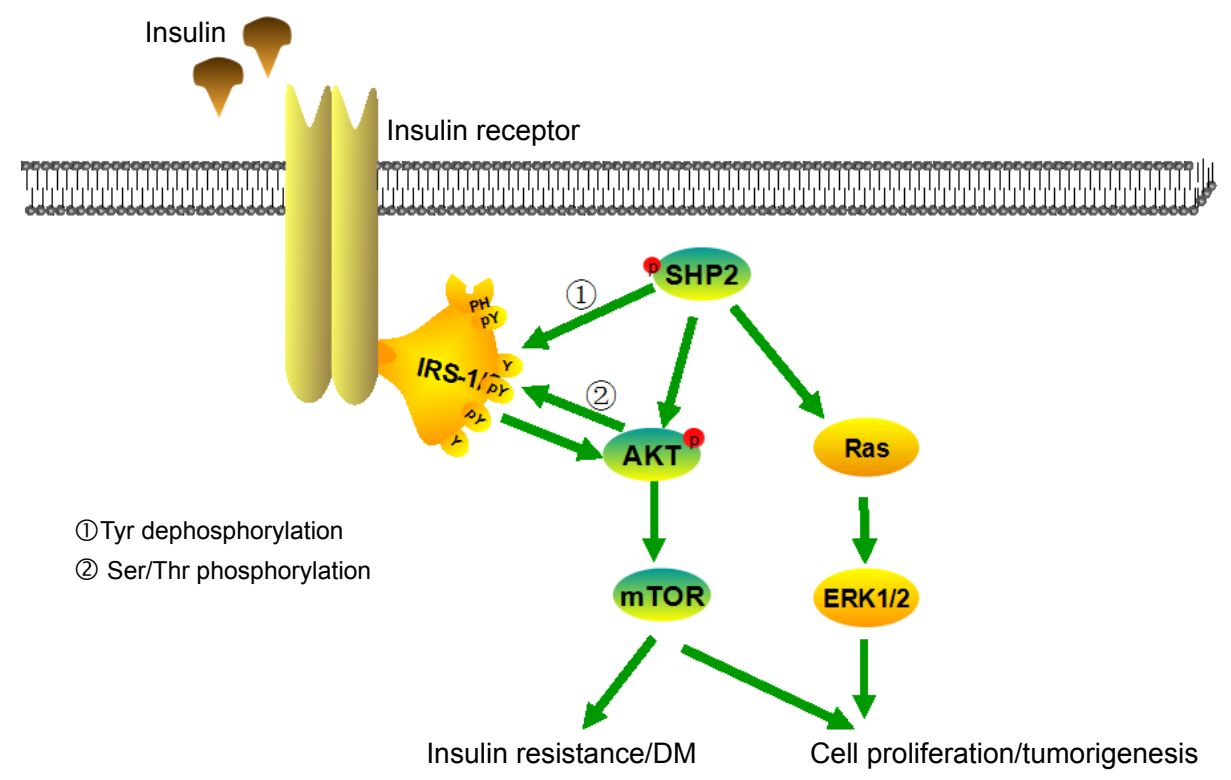

Figure 1: The role of SHP2 involved in T2DM and cancers are schematically.

to discover new potential therapeutic targets in the prevention and treatment of cancers.

\section{Evaluation of the Hypothesis}

\section{The role of PI3K/Akt/mTOR signaling pathway in T2DM}

It has been generally accepted that the understanding of insulin resistance is the key to the prevention and treatment of T2DM. However, the mechanism underlying insulin resistance is not entirely clear. Large number of studies in recent years has established the central role of $\mathrm{PI} 3 \mathrm{~K} / \mathrm{Akt} / \mathrm{mTOR}$ signaling in numerous cellular processes including metabolism, growth, survival, and motility. As a ser/thr protein kinase, Akt could enhance the phosphorylation of IRS$1 / 2$ and hamper the insulin-induced signal transdution. Um et al. [40] reported that mTOR-raptor complex, also called mammalian Target of Rapamycin Complex 1 (mTORC1), and its downstream target S6 Kinase 1 (S6K1) mediate nutrient-induced insulin resistance by down regulating insulin receptor substrate proteins with subsequent reduction in Akt phosphorylation. Fraenke et al. [41] and Xie et al. [42] also demonstrated that mTOR played a critical role in betacell adaptation to hyperglycemia. Chronic inhibition of mTOR with rapamycin augments insulin resistance, beta cell dysfunction, and death. These data strongly suggest that aberrant activation PI3K/AKT/ mTOR pathway plays a vital role in pathogenesis and progression of T2DM $[43,44]$.

\section{The role of Ras/Erk signaling pathway in cancers}

The Ras family of GTPases (HRas, NRas and KRas) comprises of proteins that are highly conserved across species and play key roles in numerous basic cellular functions, including control of proliferation, differentiation, and apoptosis. Under normal physiological conditions, Ras/Erk activation is transient. However, excessive or sustained activation of Ras/Erk signaling pathway has been found in patients with a wide variety of cancers [45-47], suggesting the significant role of the Ras/Erk signaling pathway in cancer initiation and promotion. So far, several different mechanisms have been explored to account for the abnormal activation of Ras signaling pathways in tumorigenesis, including mutations in Ras, loss of GAP proteins, overexpression of
RTKs (such as EGFR), and also abnormal phosphorylation of tyrosine phosphatase upstream of Ras/Erk [48]. Besides, there is a cross-talk and complex feedback loop between Ras/Erk and PI3K/Akt/mTOR pathways [49,50], thus combined inhibition of these two pathways has been suggested as a therapeutic strategy in treatment of cancers [51,52].

Shp2 is upstream of Ras and PI3K pathways and plays a important role in both insulin resistance and carcinogenesis

SHP2, a ubiquitous tyrosine phosphatase, is thought to be inactive by forming intramolecular folding without stimulations, whereas it becomes active when the $\mathrm{N}$-terminal SH2 domains bind to phosphorylated molecules, including Grb2-associated binder (Gab), IRS-1 and etc., by forming an open conformation. Shp2 was reported to be a modulator that prolongs the activation of Erk [18,20,22], which suggests its role in carcinogenesis and development of several kinds of malignancies. Furthermore, Lima et al. [36] reported that insulininduced IRS-1/SHP2 complex was associated with insulin resistance and played a role in the control of AKT phosphorylation in an animal model. Princen et al. [34] demonstrated that SHP2 deficiency led to up regulation of PI3K/Akt pathways and insulin resistance in cardiomyocytes. Zhang et al. [39] also reported that SHP2 acted as a signal coordinator in beta-cells, orchestrating multiple pathways including PI3K/Akt and Ras/Erk to control insulin biosynthesis to maintain glucose homeostasis. Besides, a recent review focusing on insulin resistance also highlighted the role and therapeutic potential of SHP2 in the control of insulin action and glucose metabolism [53].

Taken together, these abovementioned results strongly prompt us to consider that: on one hand, SHP2 is involved in carcinogenesis and development of cancers mainly through Ras/Erk pathway. On the other hand, SHP2 also plays a significant role in insulin resistance of T2DM via PI3K/Akt/mTOR pathway. Importantly, Ras/Erk pathway can have a cross-talk with PI3K/Akt/mTOR one. Based on these findings, we conclude that SHP2 might present a key molecule linked both T2DM and cancers.

To verify our hypothesis, the following issues will be carefully and comprehensively addressed for some certain cancer such as lung cancer: 
1) Animal model (mouse) for T2DM should be firstly established.

2) Inhibition of SHP2 to test the activation of PI3K/AKT/mTOR and Ras/Erk between T2DM and T2DM-SHP2 ${ }^{\text {low }}$ groups.

3) Xenograft tumor experiments to compare the tumor formation between T2DM and T2DM-SHP2 ${ }^{\text {low }}$ groups (non-small cell lung cancer A549 cell line will used) in order to examine the involvement of the activation of PI3K/AKT/mTOR and Ras/ Erk.

4) Large-scale prospective study should be performed to test the expression of SHP2 in T2DM patients and the relationship between SHP2 expression and incidence of lung cancer. According to our speculation, these T2DM patients who have higher expression of SHP2 may also be suffered from higher risk of lung cancer.

\section{Consequences of the Hypothesis and Discussion}

Although a growing number of epidemiological studies suggest that T2DM may be associated with an increased risk of certain cancers, no paper up to date, to our knowledge, has elucidated the underlying mechanism linked both T2DM and cancers. In the present paper, we assume that SHP2 may present a key molecule linked both T2DM and cancers via regulating Ras/Erk and PI3K/AKT/mTOR signaling pathway. Further and comprehensive investigation of SHP2 may provide a new insight into the molecular mechanism linked both T2DM and cancers, thus help us to discover new potential therapeutic targets in the prevention and treatment of cancers. Although some questions have not been explained completely, there are still enough evidence for us to hypothesize that SHP2 may be a key target linked T2DM and cancers and a potential target for the intervention and potential therapy for cancers.

\section{Conflict of Interest}

All authors declare no conflicts of interest.

\section{Acknowledgement}

This work was supported by grants from the National Natural Science Foundation of China (NFSC, Grant no. 81071913). We also appreciate Professor Xia Zhang in the Institute of Pathology for his critical comments and English editing.

\section{References}

1. Morrison M (2012) Pancreatic cancer and diabetes. Adv Exp Med Biol 771: 229-239

2. Yang WS, Shu XO, Gao J, Li HL, Cai H, et al. (2013) Prospective evaluation of type 2 diabetes mellitus on the risk of primary liver cancer in Chinese men and women. Ann Oncol 24: 1679-1685.

3. Lee MY, Lin KD, Hsiao PJ, Shin SJ (2012) The association of diabetes mellitus with liver, colon, lung, and prostate cancer is independent of hypertension, hyperlipidemia, and gout in Taiwanese patients. Metabolism 61: 242-249.

4. Luo W, Cao Y, Liao C, Gao F (2012) Diabetes mellitus and the incidence and mortality of colorectal cancer: a meta-analysis of 24 cohort studies. Colorectal Dis 14: 1307-1312.

5. Boyle P, Boniol M, Koechlin A, Robertson C, Valentini F, et al. (2012) Diabetes and breast cancer risk: a meta-analysis. Br J Cancer 107: 1608-1617.

6. Xu X, Wu J, Mao Y, Zhu Y, Hu Z, et al. (2013) Diabetes mellitus and risk of bladder cancer: a meta-analysis of cohort studies. PLoS One 8: e58079.

7. Chen YL, Cheng KC, Lai SW, Tsai IJ, Lin CC, et al. (2013) Diabetes and risk of subsequent gastric cancer: a population-based cohort study in Taiwan. Gastric Cancer 16: 389-396.

8. Friberg E, Orsini N, Mantzoros CS, Wolk A (2007) Diabetes mellitus and risk of endometrial cancer: a meta-analysis. Diabetologia 50: 1365-1374.

9. Hochgräfe F, Zhang L, O'Toole SA, Browne BC, Pinese M, et al. (2010)
Tyrosine phosphorylation profiling reveals the signaling network characteristics of Basal breast cancer cells. Cancer Res 70: 9391-9401.

10. Castoria G, Giovannelli P, Lombardi M, De Rosa C, Giraldi T, et al. (2012) Tyrosine phosphorylation of estradiol receptor by Src regulates its hormonedependent nuclear export and cell cycle progression in breast cancer cells. Oncogene 31: 4868-4877.

11. Chellappa K, Jankova L, Schnabl JM, Pan S, Brelivet Y, et al. (2012) Src tyrosine kinase phosphorylation of nuclear receptor HNF4a correlates with isoform-specific loss of HNF4a in human colon cancer. Proc Natl Acad Sci U S A 109: 2302-2307.

12. Zhu Y, Park J, Hu X, Zheng K, Li H, et al. (2010) Control of oligodendrocyte generation and proliferation by SHP2 protein tyrosine phosphatase. Glia 58: 1407-1414.

13. Li L, Modi H, McDonald T, Rossi J, Yee JK, et al. (2011) A critical role for SHP2 in STAT5 activation and growth factor-mediated proliferation, survival, and differentiation of human CD34+ cells. Blood 118: 1504-1515.

14. Huang YS, Cheng CY, Chueh SH, Hueng DY, Huang YF, et al. (2012) Involvement of SHP2 in focal adhesion, migration and differentiation of neural stem cells. Brain Dev 34: 674-684.

15. Kuo E, Park DK, Tzvetanova ID, Leiton CV, Cho BS, et al. (2010) Tyrosine phosphatases Shp1 and Shp2 have unique and opposing roles in oligodendrocyte development. J Neurochem 113: 200-212.

16. Takahashi A, Tsutsumi R, Kikuchi I, Obuse C, Saito $Y$, et al. (2011) SHP2 tyrosine phosphatase converts parafibromin/Cdc73 from a tumor suppressor to an oncogenic driver. Mol Cell 43: 45-56

17. Bard-Chapeau EA, Li S, Ding J, Zhang SS, Zhu HH, et al. (2011) Ptpn11/Shp2 acts as a tumor suppressor in hepatocellular carcinogenesis. Cancer Cell 19: 629-639.

18. Aceto N, Sausgruber N, Brinkhaus H, Gaidatzis D, Martiny-Baron G, et al (2012) Tyrosine phosphatase SHP2 promotes breast cancer progression and maintains tumor-initiating cells via activation of key transcription factors and a positive feedback signaling loop. Nat Med 18: 529-537.

19. Li S, Hsu DD, Wang H, Feng GS (2012) Dual faces of SH2-containing proteintyrosine phosphatase Shp2/PTPN11 in tumorigenesis. Front Med 6: 275-279.

20. Dance M, Montagner A, Salles JP, Yart A, Raynal P (2008) The molecular functions of Shp2 in the Ras/Mitogen-activated protein kinase (ERK1/2) pathway. Cell Signal 20: 453-459.

21. Sampaio C1, Dance M, Montagner A, Edouard T, Malet N, et al. (2008) Signal strength dictates phosphoinositide 3-kinase contribution to Ras/extracellular signal-regulated kinase 1 and 2 activation via differential Gab1/Shp2 recruitment: consequences for resistance to epidermal growth factor receptor inhibition. Mol Cell Biol 28: 587-600.

22. Zhou XD, Agazie YM (2008) Inhibition of SHP2 leads to mesenchymal to epithelial transition in breast cancer cells. Cell Death Differ 15: 988-996.

23. Zhou X, Coad J, Ducatman B, Agazie YM (2008) SHP2 is up-regulated in breast cancer cells and in infiltrating ductal carcinoma of the breast, implying its involvement in breast oncogenesis. Histopathology 53: 389-402.

24. Zhou X, Agazie YM (2009) Molecular mechanism for SHP2 in promoting HER2induced signaling and transformation. J Biol Chem 284: 12226-12234.

25. Tang C, Zhou X, Yang H, Wang Q, Zhang R (2010) [Expression and its clinical significance of SHP2 in non-small cell lung cancer]. Zhongguo Fei Ai Za Zhi 13: 98-101.

26. Tang C, Luo D, Yang H, Wang Q, Zhang R, et al. (2013) Expression of SHP2 and related markers in non-small cell lung cancer: a tissue microarray study of 80 cases. Appl Immunohistochem Mol Morphol 21: 386-394.

27. Zhang ZJ, Zheng ZJ, Shi R, Su Q, Jiang Q, et al. (2012) Metformin for liver cancer prevention in patients with type 2 diabetes: a systematic review and meta-analysis. J Clin Endocrinol Metab 97: 2347-2353.

28. Zhang ZJ, Zheng ZJ, Kan H, Song Y, Cui W, et al. (2011) Reduced risk of colorectal cancer with metformin therapy in patients with type 2 diabetes: a meta-analysis. Diabetes Care 34: 2323-2328.

29. Tseng CH (2012) Diabetes, metformin use, and colon cancer: a populationbased cohort study in Taiwan. Eur J Endocrinol 167: 409-416.

30. Thakkar B, Aronis KN, Vamvini MT, Shields K, Mantzoros CS (2013) Metformin and sulfonylureas in relation to cancer risk in type II diabetes patients: a metaanalysis using primary data of published studies. Metabolism 62: 922-934. 
Citation: Luo H, Tang C, Yang X, Zhou X (2014) The Tyrosine Phosphatase SHP2: A Key Molecule Linked both Type 2 Diabetes and Cancers? Med chem 4: 435-438. doi:10.4172/2161-0444.1000175

31. Sinnett-Smith J, Kisfalvi K, Kui R, Rozengurt E (2013) Metformin inhibition of mTORC1 activation, DNA synthesis and proliferation in pancreatic cancer cells: dependence on glucose concentration and role of AMPK. Biochem Biophys Res Commun 430: 352-357.

32. McCarty MF (2011) mTORC1 activity as a determinant of cancer risk-rationalizing the cancer-preventive effects of adiponectin, metformin, rapamycin, and low-protein vegan diets. Med Hypotheses 77: 642-648.

33. Bifulco G, Di Carlo C, Caruso M, Oriente F, Di Spiezio Sardo A, et al. (2002) Glucose regulates insulin mitogenic effect by modulating SHP-2 activation and localization in JAr cells. J Biol Chem 277: 24306-24314.

34. Princen F, Bard E, Sheikh F, Zhang SS, Wang J, et al. (2009) Deletion of Shp2 tyrosine phosphatase in muscle leads to dilated cardiomyopathy, insulin resistance, and premature death. Mol Cell Biol 29: 378-388.

35. Giri H, Muthuramu I, Dhar M, Rathnakumar K, Ram U, et al. (2012) Protein tyrosine phosphatase SHP2 mediates chronic insulin-induced endothelial inflammation. Arterioscler Thromb Vasc Biol 32: 1943-1950.

36. Lima MH, Ueno M, Thirone AC, Rocha EM, Carvalho CR, et al. (2002) Regulation of IRS-1/SHP2 interaction and AKT phosphorylation in animal models of insulin resistance. Endocrine 18: 1-12.

37. Miyakawa Y, Rojnuckarin P, Habib T, Kaushansky K (2001) Thrombopoietin induces phosphoinositol 3-kinase activation through SHP2, Gab, and insulin receptor substrate proteins in BAF3 cells and primary murine megakaryocytes. J Biol Chem 276: 2494-2502.

38. Müssig K, Staiger H, Fiedler H, Moeschel K, Beck A, et al. (2005) Shp2 is required for protein kinase C-dependent phosphorylation of serine 307 in insulin receptor substrate-1. J Biol Chem 280: 32693-32699.

39. Zhang SS, Hao E, Yu J, Liu W, Wang J, et al. (2009) Coordinated regulation by Shp2 tyrosine phosphatase of signaling events controlling insulin biosynthesis in pancreatic beta-cells. Proc Natl Acad Sci U S A 106: 7531-7536.

40. Um SH, D'Alessio D, Thomas G (2006) Nutrient overload, insulin resistance, and ribosomal protein S6 kinase 1, S6K1. Cell Metab 3: 393-402.

41. Fraenkel M, Ketzinel-Gilad M, Ariav Y, Pappo O, Karaca M, et al. (2008) mTOR inhibition by rapamycin prevents beta-cell adaptation to hyperglycemia and exacerbates the metabolic state in type 2 diabetes. Diabetes 57: 945-957.

42. Xie J, Herbert TP (2012) The role of mammalian target of rapamycin (mTOR) in the regulation of pancreatic $\beta$-cell mass: implications in the development of type-2 diabetes. Cell Mol Life Sci 69: 1289-1304.

43. Singh S, Ramamoorthy M, Vaughan C, Yeudall WA, Deb S, et al. (2013) Human oncoprotein MDM2 activates the Akt signaling pathway through an interaction with the repressor element- 1 silencing transcription factor conferring a survival advantage to cancer cells. Cell Death Differ 20: 558-566.

44. Khan MW, Keshavarzian A, Gounaris E, Melson JE, Cheon EC, et al. (2013) $\mathrm{PI} 3 \mathrm{~K} / \mathrm{AKT}$ signaling is essential for communication between tissue-infiltrating mast cells, macrophages, and epithelial cells in colitis-induced cancer. Clin Cancer Res 19: 2342-2354.

45. Santarpia L, Lippman SM, El-Naggar AK (2012) Targeting the MAPK-RAS-RAF signaling pathway in cancer therapy. Expert Opin Ther Targets 16: 103-119.

46. Prior IA, Lewis PD, Mattos C (2012) A comprehensive survey of Ras mutations in cancer. Cancer Res 72: 2457-2467.

47. Mulholland DJ, Kobayashi N, Ruscetti M, Zhi A, Tran LM, et al. (2012) Pten loss and RAS/MAPK activation cooperate to promote EMT and metastasis initiated from prostate cancer stem/progenitor cells. Cancer Res 72: 1878-1889.

48. Castellano E, Downward J (2011) RAS Interaction with PI3K: More Than Just Another Effector Pathway. Genes Cancer 2: 261-274.

49. De Luca A, Maiello MR, D'Alessio A, Pergameno M, Normanno N (2012) The RAS/RAF/MEK/ERK and the PI3K/AKT signalling pathways: role in cancer pathogenesis and implications for therapeutic approaches. Expert Opin Ther Targets 16 Suppl 2: S17-27.

50. Huang J, Che MI, Lin NY, Hung JS, Huang YT, et al. (2014) The molecular chaperone Cosmc enhances malignant behaviors of colon cancer cells via activation of Akt and ERK. Mol Carcinog 53 Suppl 1: E62-71.

51. Slomovitz BM, Coleman RL (2012) The PI3K/AKT/mTOR pathway as a therapeutic target in endometrial cancer. Clin Cancer Res 18: 5856-5864.

52. Shimizu T, Tolcher AW, Papadopoulos KP, Beeram M, Rasco DW, et al. (2012) The clinical effect of the dual-targeting strategy involving PI3K/AKT/mTOR and RAS/MEK/ERK pathways in patients with advanced cancer. Clin Cancer Res 18: $2316-2325$.

53. Xu E, Schwab M, Marette A (2014) Role of protein tyrosine phosphatases in the modulation of insulin signaling and their implication in the pathogenesis of obesity-linked insulin resistance. Rev Endocr Metab Disord 15: 79-97. 